\title{
Method for probing the magnetic state of nanomaterials encapsulated in carbon nanotubes
}

\author{
Satoru Konabe ${ }^{a)}$ and Susumu Okada \\ Graduate School of Pure and Applied Sciences, University of Tsukuba, Tsukuba, Ibaraki 305-8571, Japan \\ and Japan Science and Technology Agency, CREST, 5 Sanbancho, Chiyoda, Tokyo 102-0075, Japan
}

(Received 14 December 2010; accepted 28 January 2011; published online 17 February 2011)

\begin{abstract}
We propose a method for optically probing the magnetic states of metallic atoms encapsulated in single-walled carbon nanotubes. The absorption spectrum is calculated by solving the BetheSalpeter equation, which includes the effects of magnetic atoms, under the tight-binding approximation. Due to the exchange interaction between excitons and polarized spins in ferromagnets, triplet excitons acquire a finite oscillator strength and can thus be excited by light. This mechanism is promising for detecting magnetic ordering of materials encapsulated in carbon nanotubes. (C) 2011 American Institute of Physics. [doi:10.1063/1.3556274]
\end{abstract}

Miniaturization and advances in electronic devices demand the development of materials that can be used as constituent elements in emerging post-silicon devices. Atomically thin nanowires of both semiconductors and metals are the most promising of these materials for practical applications. ${ }^{1}$ The ability to control the electronic states of these wires will lead to the development of devices such as nanoscale switches and spintronic and quantum devices. Carbon nanotubes (CNTs) are key materials for producing templates for ultrathin nanowires since they provide a stable quasi-one-dimensional nanospace ${ }^{2}$ that protects encapsulated materials from the environment.

Kitaura et al. recently synthesized atomically thin $\mathrm{Eu}$ nanowires in carbon nanotubes and demonstrated their stability. ${ }^{3}$ Transmission electron microscopy images reveal that Eu nanowires have interesting morphologies, which vary depending on the space provided by the nanotubes. Although their structures have been examined, their electronic properties remain undetermined. Since Eu atoms usually induce magnetism in solid and compound phases, Eu nanowires are also expected to exhibit magnetism. From both scientific and technological viewpoints, simple methods for probing the magnetic states of nanowires in nanotubes are highly desirable. Direct measurements of the magnetic susceptibility are useful since they directly detect magnetic properties. ${ }^{3}$ However, noncontact probes are very attractive for evaluating the magnetic properties of devices since the magnetic state of nanomaterials may be sensitive to external factors. Optical measurements are very promising for such probes if they can detect the magnetic ordering of encapsulated atoms.

Carbon nanotubes exhibit very unusual photoresponses due to their quasi-one-dimensional structure, which enhances the Coulomb interaction between excited carriers. ${ }^{4}$ Consequently, a photon generates a strongly bound electron-hole pair (known as an exciton) that has a binding energy of a few hundreds of $\mathrm{meV}$ in semiconducting carbon nanotubes. ${ }^{5-7}$ The exchange interaction splits exciton spin states into singlet and triplet states with different energies. ${ }^{6,8-11}$ These energetically different spin states can be used to probe the magnetic states of materials encapsulated in carbon nanotubes. Below a transition temperature, the randomly distributed

${ }^{\text {a)} E l e c t r o n i c ~ m a i l: ~ k o n a b e @ c o m a s . f r s c . t s u k u b a . a c . j p . ~}$ spins of ferromagnetic atoms become ferromagnetically ordered. These polarized spins may interact with exciton spins in nanotubes, thereby altering the exciton spin states. The crucial point is that spin-triplet excitons, which are initially dark, can be excited by magnetically ordered spins in encapsulated materials. Therefore, a peak due to triplet excitons may appear in addition to the peak due to bright spin-singlet excitons. We thus expect that the optical response will differ depending on whether carbon nanotubes contain ferromagnet atoms or not.

In this paper, we theoretically propose a method for quantitatively probing the magnetic state of atomically thin nanowires encapsulated in carbon nanotubes based on calculations of the optical responses of semiconducting carbon nanotubes performed by solving the tight-binding BetheSalpeter equation. ${ }^{6,12}$ The influences of encapsulated magnetic elements are introduced via the simple exchange interaction between the spins of excitons and the spins of magnetic atoms. The calculated absorption spectra exhibit a peak that originates from the coupling between a singlet bright exciton and triplet dark excitons. Based on this finding, we propose a method for detecting the magnetic properties of encapsulated magnetic elements in carbon nanotubes that employs an optical spectroscopic technique.

To discuss the effects of magnetic elements on excitons in nanotubes, we solve the Bethe-Salpeter equation that includes the exchange interaction between excitons and a ferromagnet. This exchange interaction can be modeled by the following Hamiltonian:

$$
\mathcal{H}_{\text {mag }}=-\sum_{\mathbf{r}}\left[J_{c} \boldsymbol{s}_{c}-J_{v} \boldsymbol{s}_{v}\right] \cdot S(\dot{\mathbf{r}})
$$

where $\boldsymbol{s}_{c(v)} \equiv \Sigma_{k, k^{\prime}} \Sigma_{\sigma, \sigma^{\prime}} c_{k, \sigma}^{\dagger} \boldsymbol{\sigma}_{\sigma, \sigma^{\prime}} c_{k^{\prime}, \sigma^{\prime}}$ is the spin of conduction (valence) electrons and $\boldsymbol{S}$ is the spin of the localized state. $\boldsymbol{\sigma}$ are the Pauli matrices. The explicit form of the exchange integrals $J_{c}\left(J_{v}\right)$ in Eq. (1) depend on the materials being considered. These two exchange integrals generally have different values. When the spins of ferromagnetic atoms are ferromagnetically ordered below the phase transition temperature, Eq. (1) can be treated as 


$$
\mathcal{H}_{\text {mag }}=-N\left(J_{c} s_{z}^{c}-J_{v} s_{z}^{v}\right)\left\langle S_{z}\right\rangle,
$$

where $\left\langle S_{z}\right\rangle$ is the thermal average of spins and $N$ is the number of magnetic ions in a ferromagnet. The polarized spins behave as an effective magnetic field to excitons,

$$
\mathcal{H}_{\text {mag }}=g \mu_{B} s_{z}^{c} H_{z}^{c}-g \mu_{B} s_{z}^{v} H_{z}^{v},
$$

where the effective field $H_{z}^{c(v)}$ is defined as $H_{z}^{c(v)} \equiv-J_{c(v)} M /\left(g \mu_{B}\right)^{2}$, where $\mu_{B}$ is the Bohr magneton, $g$ $=2$ is the g-factor, and $M \equiv-N g \mu_{B}\left\langle S_{z}\right\rangle$ is the magnetization. Equation (3) indicates that an electron in the conduction band and a hole in the valence band have different Zeeman energies due to the different exchange integrals. Accordingly, the spin degeneracy is broken in the presence of the exchange so that singlet and triple excitons can mix. Hereafter, we assume that the spins in a ferromagnet are saturated, so we set $\left\langle S_{z}\right\rangle=S(=7 / 2)$ for Eu atoms. ${ }^{3}$

The exciton states are obtained by solving the BetheSalpeter equation, ${ }^{5,6,11,12}$

$$
\left(\varepsilon_{k_{c}}-\varepsilon_{k_{v}}\right) Z_{k}^{n}+\sum_{k^{\prime}} K_{k, k^{\prime}} Z_{k^{\prime}}^{n}=E^{n} Z_{k}^{n},
$$

where $Z_{k}^{n}$ and $E^{n}$ are, respectively, the exciton amplitude and the energy of the $n$-state. $K_{k, k^{\prime}}$ is the kernel, which consists of exchange $K_{k, k^{\prime}}^{x}$, screened-direct $K_{k, k^{\prime}}^{d}$, and ferromagnetic exchange terms,

$$
\begin{aligned}
& \left\langle\uparrow \uparrow\left|K_{k, k^{\prime}}\right| \uparrow \uparrow\right\rangle=K_{k, k^{\prime}}^{d}+K_{k, k^{\prime}}^{x}+N J\left\langle S_{z}\right\rangle \delta_{k, k^{\prime}}, \\
& \left\langle\uparrow \downarrow\left|K_{k, k^{\prime}}\right| \uparrow \downarrow\right\rangle=K_{k, k^{\prime}}^{d}+N\left(J_{c}+J_{v}\right)\left\langle S_{z}\right\rangle \delta_{k, k^{\prime}}, \\
& \left\langle\downarrow \uparrow\left|K_{k, k^{\prime}}\right| \downarrow \uparrow\right\rangle=K_{k, k^{\prime}}^{d}-N\left(J_{c}+J_{v}\right)\left\langle S_{z}\right\rangle \delta_{k, k^{\prime}}, \\
& \left\langle\downarrow \downarrow\left|K_{k, k^{\prime}}\right| \downarrow \downarrow\right\rangle=K_{k, k^{\prime}}^{d}+K_{k, k^{\prime}}^{x}-N J\left\langle S_{z}\right\rangle \delta_{k, k^{\prime}}, \\
& \left\langle\uparrow \uparrow\left|K_{k, k^{\prime}}\right| \downarrow \downarrow\right\rangle=K_{k, k^{\prime}}^{x}=\left\langle\downarrow \downarrow\left|K_{k, k^{\prime}}\right| \uparrow \uparrow\right\rangle=K_{k, k^{\prime}}^{x},
\end{aligned}
$$

where $J \equiv J_{c}-J_{v}$. The quasiparticle energy $\varepsilon_{\mathbf{k}_{c}}$ and the quasihole energy $\varepsilon_{\mathbf{k}_{v}}$ are calculated by applying the randomphase approximation., ${ }^{5,11,12}$ For the Coulomb potential between $\pi$ orbitals, we employed the Ohno potential $V(\mathbf{r})$ $=U / \kappa \sqrt{\left[\left(4 \pi \epsilon_{0} / e^{2}\right) U|\mathbf{r}|\right]^{2}+1}$, with $U=11.3 \mathrm{eV} \cdot{ }^{6,11,12}$ The dielectric function $\kappa=1.8$ for vacuum ${ }^{13}$ incorporated screening effects due to $\sigma$ bands and the surrounding environment. The calculations were performed under the tight-binding approximation taking nearest-neighbor hopping of $2.7 \mathrm{eV}$ into account.

Using the exciton state obtained from Eq. (4), the absorption spectra are calculated using the following equation:

$$
\alpha(\omega)=\frac{\pi \hbar^{3}}{e^{2} \varepsilon_{0} c} \sum_{n}\left|\sum_{k} \frac{Z_{k}^{n} D_{k}}{E^{n}}\right|^{2} \delta\left(\hbar \omega-E^{n}\right),
$$

where $D_{k}=\left\langle k_{c}|z| k_{v}\right\rangle$ is the dipole matrix element between the conduction $\left(\left|k_{c}\right\rangle\right)$ and valence $\left(\left|k_{v}\right\rangle\right)$ bands. The light polarization axis is taken to be parallel to the nanotube axis (i.e., the $z$-axis) because this polarization configuration gives the largest optical absorption of a laser beam.

We first present the calculated absorption spectra. The spectra in Fig. 1 are clearly sensitive to the ferromagnet. For finite values of $N J\left(=N J_{c}-N J_{v}\right)$, a peak appears in addition to

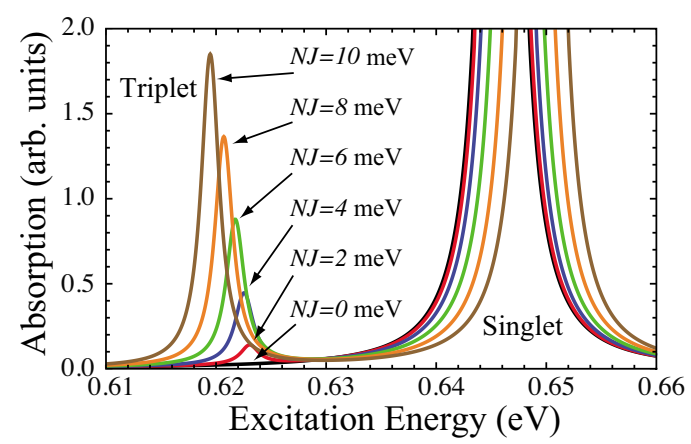

FIG. 1. (Color online) Absorption spectra of $(20,0)$ carbon nanotubes for $N J=0,2,4,6,8$, and $10 \mathrm{meV}$. Broadening is set to $1 \mathrm{meV}$.

the singlet exciton peak. The energy of the peak is about $0.03 \mathrm{eV}$ lower than that of the singlet peak, which indicates that the peak is associated with adsorption of a triplet exciton. The peak is due to the triplet exciton acquiring a finite oscillator strength. The peak intensity increases monotonically with increasing number of magnetic elements $N$ and increasing exchange interaction $J$.

Furthermore, both the singlet and triplet peaks shift with increasing $N J$. Figure 2 shows the exciton energies as a function of the exchange energy, $N J$. With increasing exchange energy, the singlet (triplet) exciton is redshifted (blueshifted) due to mixing of the singlet and triplet excitons (see below).

It is important to discuss the mechanism of the finite oscillator strength of the triplet excitons. Spin degeneracy is not broken without the Zeeman coupling expressed in Eq. (3), which is associated with the exchange interaction between excitons and polarized spins in a ferromagnet. Therefore, without the Zeeman coupling, the exciton spin states are classified according to whether they are singlet or triplet spin states and they produce the singlet peak in the absorption spectra. However, in the presence of Zeeman coupling (3), the exciton spin states cannot be classified according to whether they are the singlet and triplet spin states, which implies that singlet and triplet excitons mix with each other. Equations (5) and (9) show that, of the three degenerate triplet states, singlet excitons, $(|\uparrow \uparrow\rangle+|\downarrow \downarrow\rangle) / \sqrt{2}$, can couple only with a triplet exciton, $(|\uparrow \uparrow\rangle-|\downarrow \downarrow\rangle) / \sqrt{2}$. The spin projection in the $z$-direction of the mixed triplet state is zero $\left(s_{z} \equiv s_{z}^{c}-s_{z}^{v}\right.$ $=0)$. Singlet and triplet mixing occurs only if the exchange integrals for electrons and holes are different (i.e., $J \neq 0$ ). The actual values of the exchange integrals depend on the encapsulated material.

Next, we discuss the $J$-dependence of the spectral weight of the absorption. The spectral weight transfers from the main peak of the singlet exciton state to the satellite peak of

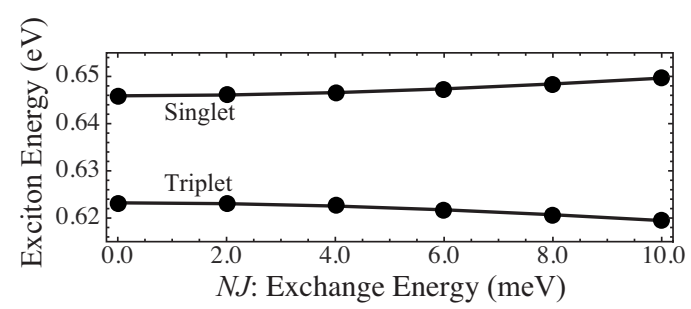

FIG. 2. Exciton energies as a function of the exchange energy. The upper (lower) series is for a spin-singlet (triplet) exciton. For triplet excitons, only the energy of the triplet exciton that couples to the singlet exciton is shown. 


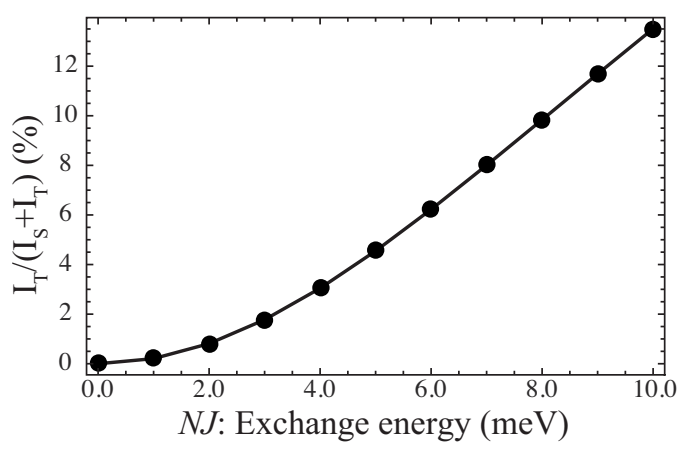

FIG. 3. Exchange integral dependence of the weight transfer from the singlet exciton to the triplet exciton of $(20,0)$ CNTs.

the triplet states according to $I_{\mathrm{T}} /\left(I_{\mathrm{S}}+I_{\mathrm{T}}\right)$, where $I_{\mathrm{S}(\mathrm{T})}$ $\equiv\left|\sum_{k} Z_{k}^{\mathrm{S}(\mathrm{T})} D_{k} / E^{\mathrm{S}(\mathrm{T})}\right|^{2}$. Here, $E^{\mathrm{S}(\mathrm{T})}$ and $Z_{k}^{\mathrm{S}(\mathrm{T})}$ are the singlet (triplet) exciton energy and amplitude, respectively. Figure 3 shows the calculated weight transfer, $I_{\mathrm{T}} /\left(I_{\mathrm{S}}+I_{\mathrm{T}}\right)$, as a function of $J$. As the product of the exchange integral and the number of magnetic elements $N J$ increases, the weight transfer increases quadratically since mixing between singlet and triple excitons is enhanced. This result demonstrates that optical spectroscopic measurements can detect not only the magnetic state but also the structural properties of nanomaterials in carbon nanotubes.

Finally, we discuss the relation to the optical response of excitons interacting with paramagnetically localized spins. ${ }^{14}$ As shown above, the interaction with the ferromagnet produces a satellite peak due to the triplet exciton in absorption spectra. When there are paramagnetically localized spins that couple to excitons, the excited spin-singlet excitons are scattered by those spins. Consequently, a satellite peak of the triplet exciton also appears, similar to the ferromagnetic case. However, the spectral shape for the interaction between excitons and a ferromagnet is quite different from that for the paramagnetic case. For the ferromagnetic case, a symmetric triplet exciton peak appears (see Fig. 1), whereas an asymmetric peak is obtained for the paramagnetic case. ${ }^{14}$ In the latter case, singlet bright excitons are scattered to triplet exciton states with finite center-of-mass momenta (i.e., it involves a band of the triplet exciton). Consequently, the spectral shape associated with the triplet states becomes asymmetric. This difference in the spectral shapes may be useful for distinguishing magnetic ordering of encapsulated materials.

A related phenomenon, namely, activation of the triplet exciton, has been observed in recent photocurrent measurements ${ }^{15}$ in a EuS-coated carbon nanotube. In this case, photocurrent spectroscopy reveals the exchange interaction between excitons in carbon nanotubes and polarized spins in the ferromagnetic EuS. Our calculation, which is based on a simple exchange interaction model of excitons with a ferromagnet [Eq. (2)], also explains this experimental observation of a photocurrent from triplet dark excitons. From our calculations, the photoactivated triplet exciton has a spin projection of $s_{z}=0$. Therefore, if our model [i.e., Eq. (2)] is valid, ferromagnet-coated carbon nanotubes may not be suitable for polarized spin injection.

This work was supported by the Japan Science and Technology Agency (CREST) and a Grant-in-Aid for Scientific Research from the Ministry of Education, Culture, Sports, Science and Technology (MEXT) of Japan.

${ }^{1}$ W. Lu and C. M. Lieber, Nature Mater. 6, 841 (2007).

${ }^{2}$ S. Okada, S. Saito, and A. Oshiyama, Phys. Rev. Lett. 86, 3835 (2001).

${ }^{3}$ R. Kitaura, R. Nakanishi, T. Saito, H. Yoshikawa, K. Awaga, and H. Shinohara, Angew. Chem. Int. Ed. 48, 8298 (2009).

${ }^{4}$ A. Jorio, G. Dresselhaus, and M. S. Dresselhaus, Carbon Nanotubes: Advanced Topics in the Synthesis, Structure, Properties and Applications (Springer, Heidelberg, 2008).

${ }^{5}$ T. Ando, J. Phys. Soc. Jpn. 66, 1066 (1997).

${ }^{6}$ V. Perebeinos, J. Tersoff, and P. Avouris, Phys. Rev. Lett. 92, 257402 (2004).

${ }^{7}$ C. D. Spataru, S. Ismail-Beigi, L. X. Benedict, and S. G. Louie, Phys. Rev. Lett. 92, 077402 (2004).

${ }^{8}$ H. Zhao and S. Mazumdar, Phys. Rev. Lett. 93, 157402 (2004).

${ }^{9}$ V. Perebeinos, J. Tersoff, and P. Avouris, Nano Lett. 5, 2495 (2005).

${ }^{10}$ C. D. Spataru, S. Ismail-Beigi, R. B. Capaz, and S. G. Louie, Phys. Rev. Lett. 95, 247402 (2005).

${ }^{11}$ T. Ando, J. Phys. Soc. Jpn. 75, 024707 (2006).

${ }^{12}$ J. Jiang, R. Saito, G. G. Samsonidze, A. Jorio, S. G. Chou, G. Dresselhaus, and M. S. Dresselhaus, Phys. Rev. B 75, 035407 (2007).

${ }^{13}$ R. B. Capaz, C. D. Spataru, S. Ismail-Beigi, and S. G. Louie, Phys. Status Solidi B 244, 4016 (2007).

${ }^{14}$ S. Konabe and S. Okada (unpublished).

${ }^{15}$ A. D. Mohite, T. S. Santos, J. S. Moodera, and B. W. Alphenaar, Nat. Nanotechnol. 4, 425 (2009). 